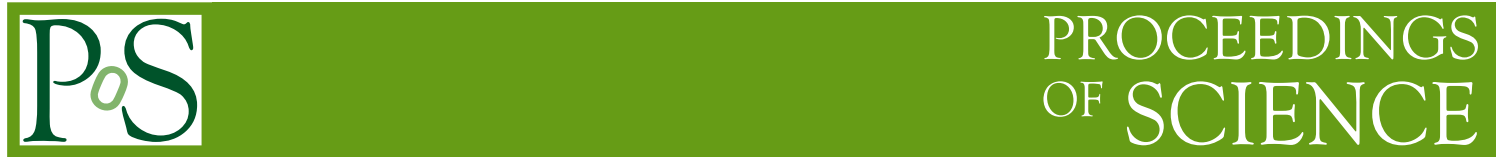

\title{
Black Holes and the Strong CP Problem
}

\section{John Swain*}

Department of Physics, Northeastern University, Boston, MA 02115, USA

E-mail: john.swainecern.ch

The strong $\mathrm{CP}$ problem is that $\mathrm{SU}(3)$ gauge field instantons naturally induce a $\mathrm{CP}$ violating term in the QCD Lagrangian which is constrained by experiment to be very small for no obvious reason. We show that this problem disappears if one assumes the existence of at least one black hole somewhere in the universe. The argument is reminiscent of Dirac's argument for the quantization of charge, in which the existence of one monople anywhere in the universe suffices to require the quantization of electric charge everywhere. Comments are made regarding appearance of similar terms in spacetimes of general topology.

VIII International Workshop on the Dark Side of the Universe,

June 10-15, 2012

Rio de Janeiro, Brazil

\footnotetext{
${ }^{*}$ Speaker.
} 


\section{Introduction}

In quantum chromodynamics (QCD)[1] - the generally accepted theory of quarks and gluons - there was a prediction that there should be a light pseudoscalar particle associated with the conserved current associated with global chiral rotations of the quarks. No such meson was observed, and this was called the "U(1) problem". It was realized that the quantum effects spoiled the conservation of the quark axial current, making its divergence proportional to $\operatorname{Tr} F_{\mu \nu} F^{* \mu v}$ where $F_{\mu \nu}$ is the $S U(3)$ field strength, $F^{* \mu v}$ its Hodge dual, and the trace is taken over $S U(3)$ indices.

This divergence corresponds to a $\mathrm{CP}$-violating Lagrangian density of the form

$$
L_{C P-\text { violating }}=\frac{\theta g^{2}}{32 \pi^{2}} \operatorname{Tr} F_{\mu v} F^{* \mu \nu}
$$

where $g$ is the $S U(3)$ gauge coupling constant, and $\theta$ is a free parameter. Overall, this expression is proportional to the Pontryagin density, which on integration over spacetime is an integer topological charge representing the number $n$ of times that $S^{3}$ (considered as physical space $\mathbb{R}^{3}$ plus a point at infinity) nontrivially "winds around" $S U(3)$. The physical gauge-invariant vacuum is constructed as a superposition of states of winding number $n$, each weighted by $e^{i n \theta}$ with the sum running from $n=-\infty$ to $n=\infty$ in order to preserve gauge invariance under the "large" gauge transformations which are not continuously connected to the identity. $\theta$ is not determined by the theory, and can, in principle, take any value between 0 and $2 \pi$.

When the weak interactions and quark masses are included, $\theta$ is shifted by an amount $\arg (\operatorname{det}(m))$ where $m$ is the quark mass matrix, but the basic form of the expression remains the same and unless the shifted $\theta$ is zero (or $\pi$, but this subtlety will not concern us here) this term leads to a (CP-violating) electric dipole moment $\left|d_{n}\right|$ for the neutron. The present upper bound $\left|d_{n}\right|<$ $2.9 \times 10^{-26} e \cdot \mathrm{cm}$, where $e$ is magnitude of the electron charge[2], implies $\theta<10^{-9}$. The puzzle of why $\theta$ is so small is the "strong $\mathrm{CP}$ problem".

A wide variety of solutions have been proposed, generally involving new physics. Many postulate particles called axions[1] associated with an additional $U(1)$ symmetry which can be used to rotate $\theta$ to zero. These have not been observed and are in general quite constrained by astrophysical considerations. Other ideas include adding dimensions to the usual $3+1$ that we know [4], or making them some fractional value a little less than four [5]. Two-dimensional fundamental objects (2-branes) [6] have been considered, as have microscopic wormholes [7], hypothetical new interactions [8], new (non-axion) particles [9], supersymmetry [10], and magnetic monopoles[11]. It has also been claimed that certain choices of regularization techniques could solve the problem[12]. It has even been suggested that a staggering $10^{32}$ copies of the Standard Model could do the job [13]. It has also been argued[14] that the strong CP problem might naturally not appear at all if one simply reformulated QCD in terms of holonomies (gauge invariant traces of Wilson loops). This list of ideas and references is not meant to be complete, but rather to show that the problem has driven theorists to a wide range of quite exotic scenarios in the search for an explanation. Despite all this creativity, the strong CP problem is still generally considered unsolved.

The point of this paper is that the problem could be resolved without unobserved exotica, and without spoiling the solution of the $U(1)$ problem, if the spacetime integral of the Pontryagin den- 
sity were somehow to be zero - something I now argue will happen if one allows for the existence of even one black hole.

In elementary particle physics one usually ignores gravity, and works with quantum field theory in flat and topologically trivial spacetime. While quantum field theory in a general curved spacetime[3] is highly nontrivial, the question asked here only requires a little topology.

First let us recall where the instantons come from that lead to the strong $\mathrm{CP}$ problem[15, 16]. We look for $S U(3)$ gauge field configurations $A_{\mu}$ which go to the identity (up to a gauge transformation) at spatial infinity, with all the directions at infinity identified. These turn out to fall into topologically distinct classes labelled by elements of $\pi_{3}(S U(3))$.

For completeness, and to make clear the origin of the $\pi_{3}(S U(3))$, let us repeat the argument in more detail. Pure gauge configurations are of the form $A_{\mu}=i U^{\dagger}(x) \partial_{\mu} U(x)$ where $U(x)$ takes its values in $S U(3)$ and $x=(t, \vec{x})$. Using the gauge freedom to set $A_{0}=0$, which essentially means we consider time-independent fields, only partially fixes the gauge. If we require $U(\vec{x})=1$ as the spatial $\vec{x}$ goes to infinity in all directions this is the same as looking for maps from spatial $\mathbb{R}^{3}$ compactified at infinity (that is, $S^{3}$ ) into $S U(3)$. Instantons and correspond to homotopy classes $\left[S^{3}, S U(3)\right]$ of these maps. By definition, $\left[S^{3}, S U(3)\right]=\pi_{3}(S U(3))$ and since $\pi_{3}(S U(3))=Z$ we have homotopically distinct maps labelled by the integers, which turn out to be the very topological charges that come from the integration of the Pontryagin density in equation 1.1.

The key observation of this paper is that if we have black holes present and repeat the argument, we should replace $\left[S^{3}, S U(3)\right]$ by $[M, S U(3)]$ where $M$ is a manifold (now with boundary) created from the $S^{3}$ described above with a 3-ball bounded by a 2-sphere excised for each black hole present - effectively we are removing a set of distinct points (and balls around them) from space.

Physically, we require that the gauge fields go to the identity (up to gauge invariance) on the surfaces of black holes (as well as at infinity), in a similar spirit to reference [17] in which this condition is invoked to argue for spacetime foam as a universal regulator. Alternatively, we can regard spacetime as having punctures wherever there are black holes, corresponding to excised open balls, or even just requiring that the points at the centers of the black holes (where the curvature blows up, so these points aren't really part of a manifold) are removed. Note that we don't need to assume spacetime foam or wormholes (as have been used to argue for solutions to the strong CP problem before) and the black holes in question need not be virtual or microscopic - any astrophysical black holes (or, indeed just one) would do. In particular, $\pi_{1}(M)$ is assumed to be trivial, as is usual in considerations of the strong CP problem (and for which there is no experimental evidence to the contrary) . In many ways, this is meant to be a very conservative solution to the strong $\mathrm{CP}$ problem invoking essentially no new physics beyond what is generally known.

The scene is essentially one in which the spacelike slice of the classical background is replaced by one with some number of points removed.

Now let us consider the homotopy classes of maps $[M, S U(3)]$ from $M$ to $S U(3) . M$ is clearly simply connected $\left(\pi_{1}(M)=0\right)$, as every closed loop can be continuously shrunk to a point. If we consider possibly topologically nontrivial maps from $M$ to $S U(3)$ then the usual Postnikov construction [18] tells one that one has to consider $\pi_{2}(S U(3))$, but this is zero, and one is left with nothing to worry about except $\left[M, K\left(\pi_{1}(S U(3)), 1\right)\right]$ with $K\left(\pi_{1}(S U(3)), 1\right)$ being the relevant Eilenberg-MacLane space. 
By definition that means that $\left[M, K\left(\pi_{1}(S U(3)), 1\right)\right]=H^{1}\left(M, \pi_{1}(S U(3))\right)$. Since $M$ is simply connected, one immediately sees that this is zero, and thus all maps from $M$ to $S U(3)$ are homotopically trivial (continuously deformable to the identity). We could argue directly that it is also zero due to the fact that $S U(3)$ is simply connected and $\pi_{1}(S U(3))=0$.

If one wants to argue that the true gauge group should be $S U(3)$ with its $Z_{3}$ center divided out[19], making the first homotopy group nonzero, then the first argument given in the above paragraph still makes the case.

The integral in equation 1.1 now vanishes as the corresponding topological charge is zero, and the strong CP problem would seem to be solved. The argument is essentially the same one that one has for a local electromagnetic contribution to the divergence of the axial current, but no electromagnetic theta term since $\pi_{1}(U(1))=0$.

Clearly, analogous arguments hold for any finite-dimensional Lie group $G$ in place of $S U(3)$ since $\pi_{2}(G)$ is always zero in this case[18] and the same reasoning applies. An example is the $S U(2)$ of electroweak theory, so there is no theta term for this either.

Some care is needed if multiply connected $M$ is considered since one does not want to induce a $\theta$-like term for the $U(1)$ of electromagnetism. Such an electromagnetic $\theta$ term is absent in standard analyses since, as noted above, $\pi_{3}(U(1))=0$, and thus there are no $U(1)$ instantons to worry about.

In the case of topologically more complicated spacetimes additional gravitationally-induced $\mathrm{CP}$ violating effects may be present[20]. In particular, terms proportional to $f_{\mu v} f^{* \mu v}$ where $f$ is the electromagnetic field strength tensor and $R_{\mu \nu}^{a b} R_{a b}^{* \mu v}$ where $R$ is the spacetime curvature tensor can be present. These contributions are not usually considered part of the "strong CP problem", although it is very interesting that these terms are not obviously suppressed by powers of the Planck scale. In the case of the $R_{\mu \nu}^{a b} R_{a b}^{* \mu v}$ the spacetimes involved clearly are not of the form considered here since corresponding instantons do not refer to topologically nontrivial gauge fields over spacetime but rather topologically nontrivial spacetimes. Whether the arguments made here can be extended to this case is not obvious, but I hope to be able to return to this interersting question in future work. Of related interest is also [21] in which the suggestion is made that the usual instanton sums may need to be modified in some theories.

As this paper was being completed, I became aware of a related paper[22] by Etesi. This author considers both black and "white" holes (which it is not clear exist), finding results for $[M, S U(3)]$ which agree with those here. The claim in that paper however is not that the Pontryagin term integrates to zero, but rather that one should consider a sort of "effective homotopy" which takes into account the causal structure of the relevant spacetime and for which the corresponding homotopy classes are not trivial and the strong CP problem remains. The idea is that one should only consider homotopies whose initial and final stages can be compared by an observer in finite time. This leads to a re-appearance of the $\theta$ vacuum structure which we just got rid of, and the solution of the strong $\mathrm{CP}$ problem is based an additional assumption which is certainly not required in the usual formulation of the strong $\mathrm{CP}$ problem. In fact $\theta$ arises in a quantum mechanical superposition of states of all instanton numbers making even the meaning of a suitable observer unclear at best. Indeed the term "instanton" refers to the fact that one considers field configurations which can be thought of as at least approximately localized in time. This leads to that paper missing the key point I make here which is that even a single black hole (no "white holes" needed) suffices to make all the $S U(3)$ field configurations topologically trivial. In this way $\operatorname{Tr} F_{\mu \nu} F^{* \mu \nu}$ can still be nonzero 
locally to solve the $U(1)$ problem, while globally all the corresponding gauge field configurations are topologically trivial.

In contrast to essentially all other attempts to solve the strong $\mathrm{CP}$ problem, the approach presented here requires no modification of the standard treatment of the problem other than to include the presence of normal (indeed classical) black holes as part of the structure of spacetime. No undiscovered exotica need be invoked.

It may seem surprising that the existence of even one singular object - in this case a black hole - could have implications for elementary particle physics, but there is actually a rather old analog. Long ago in 1948, Dirac had used topological arguments to show[23] that the presence of just one magnetic monopole would require electric charge everywhere to be quantized. Here we see that, similarly, the presence of just one black hole can resolve the strong $\mathrm{CP}$ problem.

\section{Acknowledgements}

I would like to thank Kaća Bradonjić and Tom Paul for reading early drafts of this paper. I would also like to thank Egil Lillestol, Danielle Metral, Nick Ellis, and the hospitality of the CERN Latin American School of High Energy Physics 2009 in Colombia where this work was started, Luis Alvarez-Gaumé since it was during his lecture to the students that I started to think about this problem again, and of course all the students who made the school the great success that it was! I would also like to thank Gábor Etesi for email correspondence on the first draft of this paper, as well as for pointing out that while his argument in reference [22] allowed for white holes it does not require them, and for emphasizing the possible differences in considering Euclidean and Minkowsi spacetimes in calcuations. Thanks are also due to Michael Duff and Stanley Deser for email correspondence and bringing references [20] and [21] to my attention. This work was supported in part by the US National Science Foundation under grant NSF0855388.

\section{References}

[1] An excellent review covering all the QCD and strong CP problem background required for this paper is R. D. Peccei, “The Strong CP problem and axions”, Lect. Notes Phys. 741 (2008) 3-17, available online as http://arxiv.org/abs/hep-ph/0607268

[2] C. A. Baker et al., Phys. Rev. Lett. 97 (2006) 131801.

[3] See, for example, N. D. Birrell and P. C. W. Davies, "Quantum Fields in Curved Space", Cambridge University Press, 1984; S. A. Fulling, "Aspects of Quantum Field Theory in Curved Space-Time", Cambridge University Press, 1989; R. Wald, "Quantum Field Theory in Curved Spacetime and Black Hole Thermodynamics", University Of Chicago Press, 1994.

[4] S.Yu. Khlebnikov and M.E. Shaposhnikov, Phys. Lett. B203 (1988) 121; B. Grzadkowski and J. Wudka, Phys. Rev. D77 (2008) 096004; A. Fukunaga and K.I. Izawa, Phys. Lett. B562 (2003) 251; P. Q. Hung, M. Seco, A. Soddu, Nucl. Phys. B692 83; K.I. Izawa, T. Watari, and T. Yanagida, Phys. Lett. B534 (2002) 93.

[5] M. Chaichian, A. Kobakhidze, Phys. Rev. Lett. 87 (2001) 171601.

[6] G. Dvali, Phys. Rev. D74 (2006) 025019.

[7] K. Choi and R. Holman, Phys. Rev. Lett. 62 (1989) 2575; Mod. Phys. Lett. A5 (1990) 1061. 
[8] 14) S. D. H. Hsu and F. Sannino, Phys. Lett. B605 (2005) 369; G. Aldazabal , L.E. Ibanez, A.M. Uranga, JHEP 0403 (2004) 065; L. Lavoura, Phys. Lett. B391 (1997) 441.

[9] D. Chang and Wai-Yee Keung, Phys. Rev. D70 (2004) 051901.

[10] G. Hiller and M Schmalz, Phys. Lett. B514 (2001) 263; S. M. Barr, Phys. Rev. D56 (1997) 5761; Phys. Rev. D56 (1997) 1475; R. N. Mohapatra and A. Rasin, Phys. Rev. Lett. 76 (1996) 3490; R. Kuchimanchi, Phys. Rev. Lett. 76 (1996) 3486.

[11] Hua-zhong Zhang, J. Group Theor. Phys. 2 (1994) 55, Phys. Lett. B322 (1994) 374, Erratum-ibid. B326 (1994) 329.

[12] H. Banerjee, D. Chatterjee, P. Mitra, Phys. Lett. B573 (2003) 109; G. Schierholz, ICHEP 1994:0721.

[13] G. Dvali and G. Farrar, Phys. Rev. Lett. 101 (2008) 011801.

[14] H. Fort and R. Gambini, Int. J. Theor. Phys. 39 (2000) 341.

[15] R. Jackiw and C. Rebbi, Phys. Rev. Lett. 37 (1976) 172.

[16] C. Callan. R. Dashen and D. Gross, Phys. Lett. 36B (1976) 334.

[17] L. Crane and L. Smolin, Gen. Rel. Grav. 17 (1985) 1209 and Nucl. Phys. B267 (1986) 714.

[18] See, for example, "Differential Forms in Algebraic Topology", R. Bott and L. Tu, Springer Verlag, 1995.

[19] L.O’Raifeartaigh, “Group Structure of Gauge Theories”, Cambridge University Press, 1988.

[20] S. Deser, M. J. Duff, and C. J. Isham, Physics Letters 93B (1980) 419.

[21] N. Seiberg, preprint arXiv:1005.002 (2010).

[22] G. Etesi, Int. J. Theor. Phys. 46 (2007) 832.

[23] P. A. M. Dirac, Phys. Rev. 74 (1948) 817. 